\title{
EDUCACIÓN BÁSICA REGULAR CON CONTENIDO TRIBUTARIO Y SU IMPACTO EN LA CULTURA TRIBUTARIA DE LOS CONTRIBUYENTES
}

\section{BASIC EDUCATION REGULAR WITH CONTENT TAX AND ITS IMPACT ON THE CULTURE TAX OF TAXPAYERS}

\author{
Alberto Espinoza Valenzuela ${ }^{1}$ \\ aespinozav1@usmp.pe
}

Recibido: 28 de agosto 2018

Aceptado 16 febrero 2019

\section{RESUMEN}

El presente trabajo de investigación explica la importancia de la educación en la cultura tributaria de los contribuyentes con el objetivo de que puedan ser más inclusivos en el desarrollo de sus actividades y sus relaciones tributarias con la autoridad recaudadora del País. Evitando de esta forma situaciones de evasión y elusión como consecuencia de actos por acción u omisión en situaciones de desconocimiento de la normatividad del sistema de recaudación tributaria del Estado. La metodología aplicada es del tipo descriptivo explicativo de diseño transversal descriptivo comparativo. La base del análisis ha sido apreciar por qué a pesar de que actualmente se cuenta con un convenio de cooperación interinstitucional entre la Superintendencia Nacional de Aduanas y Administración Tributaria y el Ministerio de Educación para incluir en la curricula de la educación básica regular materias tributarias, este acuerdo no se concreta en acciones concretas que conlleven al logro de objetivos puntuales en este aspecto, puesto que aún persiste el problema de incumplimiento de obligaciones tributarias, por lo que es de interés, plantear acciones que coadyuven educar sobre el rol del impuesto, acciones que interesan al Estado, la administración tributaria y a la sociedad en general, para educar y fomentar cultura tributaria, siendo necesario partir desde la educación básica y recuperar la formación cívica de valores y principios. Las escuelas deben asumir el compromiso de formar ciudadanos capaces de definir, defender y hacer cumplir normas de convivencia y sobre todo ciudadanos comprometidos con el desarrollo del país.

Palabras clave: Cultura tributaria, educación básica regular, contribuyente

\section{ABSTRAC}

The present research work explains the importance of education in the tax culture of taxpayers in order that they may be more inclusive in the development of its activities and its tax relations with the tax authority of the Country. Thus preventing situations

\footnotetext{
${ }^{1}$ Docente de la Universidad de San Martín de Porres. Lima-Perú
} 
of evasion and avoidance as a result of acts by action or omission in situations of lack of knowledge of the norms of the system of tax revenue of the State.

The methodology applied is the descriptive explanation of comparative descriptive cross-sectional design. Therefore focuses on collecting opinions qualitative and quantitative of various officials of the Public Sector as well as private sector entrepreneurs to obtain relevant information about the need to promote an educational system at primary level and secondary tax content, allowing would-be citizens to develop a proper tax culture.

The basis of the analysis has been seen because while currently has an interinstitutional cooperation agreement between the National Superintendent of customs and tax administration and the Ministry of education to include in the curriculum of the regular basic education tax matters, this agreement is not concrete in concrete actions that lead to the achievement of specific objectives in this regard, since that still persists the problem of non-compliance with tax obligations so it is of interest, consider actions that contribute to educate about the role of tax, shares that interest to the State, the Administration tax and society in general, to educate and promote tax culture, being necessary starting from education Basic and regain the political education of values and principles; Schools must assume the commitment to train citizens able to define, defend and enforce norms of coexistence and above all committed citizens the country's development.

\section{Keywords: Tax culture, basic regular education, taxpayer}

\subsection{Introducción}

El número de empresas con personería jurídica individual o societaria, es un indicador de su fortaleza económica que determina las posibilidades de lograr mayores niveles de crecimiento, empleo y bienestar, así como de incrementar la riqueza local, regional y nacional. Consecuentemente con esto surge el concepto de caracterización de la CULTURA TRIBUTARIA, que plantea cómo las empresas asumen sus obligaciones tributarias en los diferentes sectores que conforman la economía de un país.

Caracterizar una empresa desde el ámbito tributario, es mirar cómo su talento humano asume procesos y obligaciones en torno a examinar previamente todos los procedimientos propuestos en lo que respecta a los aspectos tributarios antes de que sean puestos en práctica, prever el pago de sus impuestos acogiéndose a los beneficios tributarios y exenciones contempladas en la ley, manejar la auditoría y control interno que garanticen que la empresa cumpla con la obligación tributaria sustancial dentro de una política de responsabilidad social, cumpliendo además con la obligación tributaria formal. En este orden de ideas, los componentes empresa y SUNAT se interrelacionan a partir de un elemento sustancial llamado cultura tributaria.

Sin embargo, se puede observar que en nuestro país la mayoría de comerciantes son vendedores ambulantes, mecánicos, peluqueros, etcétera; emprendedores que con 
mucho esfuerzo constituyen sus empresas, pero un buen porcentaje son informales y adolecen de cultura tributaria. Presentan falta de conocimientos tributarios, afectando su conducta respecto al cumplimiento y pago de los tributos originando el desconocimiento de las obligaciones tributarias.

El origen del problema radica básicamente en la carencia de una educación cívica tributaria, que no se da en los hogares ni en los colegios. Sobre el particular, la SUNAT se ha propuesto contribuir al modelamiento de una nueva ciudadanía basada en la práctica activa de sus obligaciones y en la adopción de un mínimo de principios éticos, ofreciendo a los maestros capacitaciones sobre temas tributarios (importancia del Estado frente a la recaudación de tributos), pero estos siguen siendo muy escasos ya que aún existe la informalidad y la evasión; y existe el desconocimiento de los bienes y servicios públicos que el Estado ofrece.

No existe una conciencia tributaria del deber que tienen todos los ciudadanos de pagar voluntaria y puntualmente sus obligaciones. Existe un comportamiento inadecuado de los contribuyentes, ya que no se responsabilizan en madurar sus conocimientos tributarios para colaborar con su país, es por ello que actualmente se ve con proyección concientizar a los maestros y estudiantes en la práctica constante de valores referidos a la tributación; de manera que los colegios asuman el compromiso de formar ciudadanos capaces de definir, defender y hacer cumplir normas de convivencia, para tener un país en el cual cada peruano sienta y sepa que puede realizar sus aspiraciones personales y sociales.

Es por ello que el presente trabajo de investigación tiene como objetivo relacionar la educación básica regular con la conciencia tributaria a nivel de un mejoramiento continuo de la cultura tributaria ciudadana. De manera tal que todos los ciudadanos debemos estar capacitados en el ámbito tributario, saber dónde comienza y termina nuestra responsabilidad

Existen antecedentes de investigaciones sobre este tema habiéndose ubicado entre otros los siguientes: Valencia D., (2013)"2 concluye que es necesario desarrollar una cultura tributaria a través de una adecuada Política Tributaria, que permita a los ciudadanos concebir las obligaciones tributarias como un deber sustantivo, acorde con los valores democráticos.

Por su parte Bravo Salas, (2010) $)^{3}$ en su tesis denominada "La Política Tributaria" precisa que la educación fiscal tiene como objetivo transmitir ideas, valores y actitudes favorables a la responsabilidad fiscal y contrarios a las conductas defraudadoras. Por eso, su finalidad no es tanto facilitar contenidos académicos, sino contenidos morales.

\footnotetext{
${ }^{2}$ https://www.academia.edu/9336384/LA_POL\%C3\%8DTICA_TRIBUTARIA_Y_SU_INFLUENCI A_EN_LA_CULTURA_TRIBUTARIA_DE_LOS_COMERCIANTES_DEL_MERCADO_VINOCANCHO $\mathrm{N}$

${ }^{3}$ Bravo Salas, Felicia. Perú, La política tributaria: Experiencia en el Perú - CIAT Revista de Administración Tributaria №31 Junio 2011.
} 
De ahí que la educación fiscal deba tratarse en el aula como un tema de responsabilidad ciudadana.

Igualmente Ortiz, M., (2003) $)^{4}$ en su tesis "Evaluación de la informalidad en el Perú 1990 - 2000" menciona que el proceso de migraciones internas ha provocado una urbanización acelerada de la sociedad que terminó en la informalidad, dominándolo todo. Además de ser un fenómeno social, la economía informal tiene importantes repercusiones institucionales. De hecho, hay causas concurrentes entre ellas como la falta de preparación y conciencia tributaria desde las aulas en los estudios básicos que posteriormente genera altos costos de acceso a la formalidad, las leyes, trámites burocráticos y desconocimiento, entre otros.

Gaona, W. y Tumbaco P. (2009) $)^{5}$ señala que la cultura tributaria consiste en el nivel de conocimiento que tienen los individuos de una sociedad acerca del sistema tributario y sus funciones y que los ejes centrales para la promoción de la cultura tributaria son la información, la formación y la concientización, los cuales se articulan en torno a la razón como móvil deseable fundamental de la acción de tributar, y se incorporan en una estrategia de comunicación.

Mogollón Díaz, (2014) ${ }^{6}$ señala que el nivel de cultura tributaria en los comerciantes de la ciudad de Chiclayo en el periodo 2012 es bajo, con este estudio ha quedado evidenciado, que el contribuyente chiclayano no lleva arraigada su obligación del pago del tributo como algo inherente a su ciudadanía. La gran mayoría de los entrevistados posee una concepción negativa de la administración tributaria, considerándola ineficiente y a sus funcionarios poco o nada honrados. Contamos con ciudadanos con valores altruistas pero que no están dispuestos a cumplir con sus obligaciones tributarias porque perciben que el Estado no cumplen con sus funciones adecuadamente.

En esta misma línea Gómez Gallardo \& Macedo Buleje, $(2008)^{7}$, detallan que “(...) Por lo tanto, siendo los primeros años del desarrollo infantil, lo años fundamentales para la formación del ser humano, la educación debe estar enfocada a potenciar al máximo su desarrollo cognitivo y la formación de su conciencia moral; basados en estas teorías, el Programa de Cultura Tributaria refuerza la idea de consolidar futuros

${ }^{4}$ Ortiz, M. (2003) Tesis "Evaluación de la informalidad en el Perú 1990 - 2000". Arcor editores. En http://repositorio.uwiener.edu.pe/bitstream/handle/123456789/761/TITULO\%20\%20V\%C3\%A1squez\%20Ram\%C3\%ADrez\%2C\%20Eduardo\%20Manuel.pdf?sequence $=1$ \&isAllowed $=\mathrm{y}$

5 "La educación tributaria como medida para incrementar la recaudación fiscal en Ecuador", rescatada de la página web:

ttps://www.dspace.espol.edu.ec/bitstream/123456789/5530/1/D-38880.pdf

${ }^{6}$ Mogollón, V. (2014). Nivel de cultura tributaria en los comerciantes de la ciudad de Chiclayo en el periodo 2012 para mejorar la recaudación pasiva de la región, Chiclayo, Perú (Tesis de pregrado, Universidad Católica Santo Toribio de Mogrovejo, Chiclayo, Perú). Recuperada de http://tesis.usat.edu.pe/handle/usat/202

7 Gómez Gallardo, L.M., y Macedo Buleje, J. C. (2011). Hacia una mejor calidad de la gestión educativa peruana en el siglo XXI. Investigación Educativa Vol. 14 № 26, 39-49 julio-diciembre 2010, ISSN 1728-5852. 
ciudadanos capaces de cambiar la cultura ciudadana y tributaria tan deficiente que se vive hoy en día.

Teniendo en consideración lo anteriormente señalado y las premisas fácticas señaladas como realidad empírica, la presente investigación toma como premisa de análisis las siguientes interrogantes:

¿De qué forma la educación tributaria a niveles del sistema de educación básica regular, incide en la formación de cultura tributaria en el estudiante peruano?, $y$

¿Cómo incide el convenio de cooperación interinstitucional suscrito entre la SUNAT y el MINEDU en la contribución de la conciencia tributaria de docentes y estudiantes del Perú?

La finalidad de su elaboración a la vez toma los objetivos siguientes:

1. "De qué forma la educación tributaria a niveles del sistema de educación básica regular, incide en la formación de cultura tributaria en el estudiante peruano? y

2. ¿Cuál es el impacto del convenio de cooperación interinstitucional suscrito entre la SUNAT y el MINEDU en la contribución de la conciencia tributaria de docentes y estudiantes del Perú?".

Siendo las hipótesis a demostrar las siguientes:

1. “"La educación tributaria a niveles del sistema de educación básica regular, incide positivamente en la formación de cultura tributaria en el estudiante peruano?

2. ¿El convenio de cooperación interinstitucional suscrito entre la SUNAT y el MINEDU impacta positivamente en la contribución de la conciencia tributaria de docentes y estudiantes del Perú".

\section{METODOLOGÍA DE INVESTIGACIÓN}

La presente investigación es Descriptivo - Explicativo y Longitudinal porque se pretende conocer los resultados de la relación existente entre la educación de temas tributarios en los niveles del educación básica regular y determinar de qué manera incide en la formación de cultura tributaria. Esto implica el estudio de teorías de la educación en los niveles básicos y su vinculación con la política fiscal de un Estado; en referencia a estos aspectos se explicará el impacto que generaría en la formación de cultura. Es longitudinal, porque analizaremos a través del tiempo determinadas variables, sus relaciones entre ellas para hacer referencias respecto al cambio, sus determinantes y consecuencias. 
Hernández Sampieri (2006, págs. 104 y 108) ${ }^{8}$ sostiene que "los estudios descriptivos ofrecen la posibilidad de hacer predicciones (...), mientras que los estudios explicativos van más allá de la descripción de conceptos o fenómenos o del establecimiento de relaciones entre conceptos; es decir, están dirigidos a responder por las causas de los eventos y fenómenos físicos o sociales. Como su nombre lo indica, su interés se centra en explicar por qué ocurre un fenómeno". Por otra parte Bernal (2010, pág. 115) "afirma que la investigación descriptiva es el nivel básico de la investigación científica (...). En la investigación explicativa se analizan causas y efectos de la relación entre variables"

En cuanto al diseño de la investigación es no experimental, en tanto no se pretende manipular las variables, sino observar el desempeño de estas en diversas etapas. Al respecto Bernal (2010, pág. 145) señala que "la definición de un diseño de investigación está determinada por el tipo de investigación que va a realizarse y por la hipótesis que va a probarse durante el desarrollo de la investigación". Por su parte, Hernández Sampieri (2006, pág. 152) ${ }^{9}$ sobre el diseño no experimental sostiene que: un estudio no experimental no se genera ninguna situación, sino que se observan situaciones ya existentes, no provocadas intencionalmente en la investigación por quien la realiza. En la investigación no experimental las variables independientes ocurren y no es posible manipularlas, no se tiene control directo sobre dichas variables ni se puede influir en ellas, porque ya sucedieron, al igual que sus efectos.

En este sentido la presente investigación, es desarrollada a través de una muestra no probabilística, que está conformada por los sujetos u objetos de estudio, como los funcionarios y expertos tributarios de la Superintendencia Nacional de Aduanas y Administración Tributaria (SUNAT), docentes y catedráticos de universidades, entre otros. Se toma como población al conjunto de personas e instituciones para la cual serán validadas las conclusiones que se obtengan. Se tomará por conveniencia a los funcionarios y expertos tributaristas y del Ministerio de Educación, así como a los docentes y catedráticos de la Universidad Nacional Mayor de San Marcos como de la universidad San Martín de Porres. En ese sentido la muestra se tomará por conveniencia tal como lo amerita la presente investigación y estará conformada por funcionarios de dichas dependencias según el siguiente detalle

- 10 docentes de la Universidad Nacional Mayor de San Marcos.

- 10 docentes de la Universidad San Martín de Porres

- 05 funcionarios de la administración tributaria - SUNAT

- 05 funcionarios del Ministerio de Educación - MINEDU

8 Hernández Sampieri, Roberto Metodología de la Investigación. Mc Graw Hill, México 1997

9 lbidem 
Sobre la selección de una muestra conveniencia, Hernández Sampieri (2014, pág. $390)^{10}$ señala que "estas muestras están formadas por los casos disponibles a los cuales tenemos acceso".

En relación a las técnicas de recolección de datos estas serán el análisis documentario, como análisis textual, entrevistas y encuestas.

Por otro lado, el análisis e interpretación de la información será codificada y analizada a través de paquetes estadísticos con la finalidad de facilitar el procesamiento de datos, entre ellos se utilizará el Statistical Package for the Social Sciences (SPPS) y el Microsoft office Excel, obteniendo cuadros, gráficos, correlación y modelos matemáticos, a fin de interpretar los resultados para la probanza de la hipótesis de estudio.

\section{RESULTADOS}

En relación a la variable práctica de si la educación tributaria a niveles del sistema de educación básica regular, incide positivamente en la formación de cultura tributaria en el estudiante peruano se tuvieron los siguientes resultados

\section{Tabla 1}

Distribución de la muestra según el nivel de incidencia de la educación básica regular en la cultura tributaria del contribuyente peruano.

\begin{tabular}{lll} 
Categorías & Frecuencia & Porcentaje \\
\hline
\end{tabular}

Baja (puntaje 6-12) $\quad 2 \quad 6 \%$

Media (puntaje 13-24)

Alta (puntaje 25-30) 5 23
$77 \%$

$17 \%$

Se incluyó en el cuestionario preguntas específicas las cuales permiten determinar el nivel de incidencia de la educación básica regular en la formación de la cultura tributaria en los futuros ciudadanos del país. Para estas preguntas el puntaje mínimo es 6 y el máximo es 30, estableciéndose los grupos por cuartiles y se observó que el $77 \%$ de los encuestados opinan que la influencia es destacable pero no determinante, a diferencia del 17\% de los encuestados que opinan que la educación básica regular es definitivamente influenciable en la futura cultura tributaria de los ciudadanos. Solo el

10 Ibidem 
$6 \%$ opina que esta no influye necesariamente en la formación de la cultura tributaria de los contribuyentes.

Se puede ver las preguntas mencionadas en la tabla 2

\section{Tabla 2}

Incidencia de la educación básica regular en la cultura ciudadana de los Contribuyentes

1.- ¿La incorporación de contenidos tributarios en la currícula nacional, favorecería la formación de cultura tributaria?

2.- Los expertos de la educación sostienen que la transmisión de conocimientos en la educación básica regular, requiere un docente capacitado, entonces, si los docentes de la EBR se encuentran capacitados en contenidos tributarios, influirá favorablemente en la formación de cultura tributaria

3.- ¿La enseñanza de contenidos tributarios a niveles de la educación básica regular en los estudiantes, desarrollará una moral autónoma frente al incumplimiento tributario?

4.- Existe un convenio interinstitucional entre SUNAT y el MINEDU para el desarrollo de contenidos tributarios en los niveles de la educación básica regular, en su opinión considera que ¿El convenio no ha sido implantado de forma continua en los centros educativos, de tal forma que impacte en la formación de cultura tributaria?

5.- En su opinión, si la enseñanza de contenidos tributarios genera entre los docentes y los estudiantes un sentido de reflexión sobre el incumplimiento tributario, entonces ¿El conocimiento de temas tributarios influiría en la reducción de la evasión tributaria?

En relación a la variable práctica de si el convenio de cooperación interinstitucional suscrito entre la SUNAT y el MINEDU incide positivamente en la contribución de la conciencia tributaria de docentes y estudiantes del Perú se tuvieron los siguientes resultados:

Distribución de la muestra según el nivel de incidencia del convenio de cooperación suscrito entre el Ministerio de Educación y la SUNAT hacia la cultura tributaria del contribuyente peruano.

Categorías Frecuencia Porcentaje

Baja (puntaje 6-12)

$63 \%$

Media (puntaje 13-24) 
Igualmente para estas preguntas el puntaje mínimo es 6 y el máximo es 30, estableciéndose los grupos por cuartiles y se observó que el $63 \%$ de los encuestados opinan que si bien es importante la suscripción de este convenio a la fecha no se ha determinado su validez debido a que no viene dando resultados tangibles en la elevación de la conciencia tributaria y por ende de la cultura tributaria de los contribuyentes peruanos. Igualmente un $27 \%$ considera que puede ser importante la firma de este acuerdo para la elevación de la cultura tributaria en el país pero que debido a la ausencia de modelos prácticos de su aplicación a la fecha no se ha implementado adecuadamente. Solo un $3 \%$ opina que es imprescindible su uso como premisa de su finalidad.

Se puede ver las preguntas mencionadas en la tabla 3

Tabla $N^{\circ} 3$

El convenio de cooperación interinstitucional suscrito entre la SUNAT y el MINEDU incide positivamente en la contribución de la conciencia tributaria de docentes y estudiantes del Perú

1.- Los programas de cultura tributaria en América de Latina son desarrollados en su gran mayoría por parte de la Administración Tributaria, en el Perú existe un convenio interinstitucional donde la inclusión de tal programa es a través de la educación formal (A través del MINEDU). En ese sentido, ¿Considera de que un convenio interinstitucional es un instrumento que incide favorablemente en la formación de cultura tributaria?

2.- En su opinión, usted estaría de acuerdo que, ¿Sea la SUNAT quien lidere la implementación de los programas de cultura tributaria en los centros educativos?

3.- ¿Considera que el nivel de cumplimiento parcial y/o total del convenio desde el 2006 al 2017, ha contribuido a la formalización de los contenidos tributarios en las instituciones educativas del Perú?

4.- ¿Considera que el no haber dado cumplimiento a los compromisos del convenio entre la SUNAT y el MINEDU, ha desfavorecido alcanzar un nivel de cultura tributaria entre los años 2006 al 2017?

Teniendo como objetivo principal determinar si la educación básica regular es determinante para una adecuada conciencia tributaria, los resultados alcanzados en la investigación indican que sí existe una relación directa entre las variables de estudio, sin embargo, esto no sucede en la aplicación de las encuestas para validar si la ejecución del convenio implica asegurar de alguna forma una conciencia tributaria en la población de los contribuyentes. 
Por ello se utilizó la prueba no paramétrica de Spearman, debido a que los valores numéricos de las variables difieren de la distribución normal (según los resultados de la prueba de Kolmogorov-Smirnov), siendo los resultados los que se muestran en la tabla $\mathrm{N}^{\circ} 4$.

\section{Tabla $\mathbf{N}^{\circ} 4$}

Relación entre la educación básica regular auxiliada por un convenio entre el MINEDU y la SUNAT que conlleva a la población a tener una cultura tributaria.

Educación Básica Regular Cultura tributaria en el contribuyente

Con contenido tributario Rho de Spearman Significancia $(p=0.05)$

$\underline{1{ }^{\circ} \text { Hipótesis. }}$

A mayor educación básica

$\begin{array}{lll}\text { con contenido tributario } & 0.233 & 0.030\end{array}$

mayor cultura tributaria

$\underline{2^{\circ} \text { Hipótesis }}$

El convenio entre la SUNAT y el

$\begin{array}{lll}\text { MINEDU eleva la conciencia } & 0.101 & 0.354\end{array}$

tributaria de los contribuyentes

Fuente: Cuestionario aplicado. Elaboración propia

El análisis a través del coeficiente de correlación de Spearman, indica una correlación, baja directa y significativa entre las variables a mayor educación básica regular en materia tributaria debe existir una mayor conciencia tributaria y por ende una mayor cultura tributaria en los contribuyentes del país, tomando como premisa la representación efectuada en el análisis de los docentes universitarios y funcionarios de la SUNAT y el MINEDU ( $r h o=0.233$ y p=0.030); cabe señalar que al ser el valor de significancia de signo positivo la relación entre las variables es directa, es decir a mayor educación básica regular con contenido tributario habrá mayor conciencia tributaria en un futuro de dichos individuos como contribuyentes.

En este sentido, al aplicar el coeficiente de correlación de Spearman para verificar la relación existente se rechaza la hipótesis nula como la negación a la hipótesis de la investigación. Al respecto se ha recogido evidencias de un alto sentido de responsabilidad del alumno que ha sido formado con bases tributarias en comparación de aquel que no lo ha recibido. Esto está relacionado con lo señalado por la SUNAT en el sentido de que si bien la educación con contenido tributario no ha sido el camino concreto para un cambio profundo en el comportamiento de los peruanos como 
contribuyentes, este nivel de educación debe ser un paso importante para despertar la conciencia tributaria y formar una cultura tributaria que lo caracterice.

Por otro lado al aplicar el coeficiente de correlación de Spearman para validar la hipótesis referida a la viabilidad entre el convenio entre la SUNAT y el MINEDU que eleve la conciencia tributaria, conforme a los resultados $((p=0.354$ Mayor a $\alpha=0.05)$ no se rechaza la hipótesis nula toda vez que se asume no dependiente la variable que define la conciencia tributaria con la cultura tributaria con la necesidad de implementar un convenio entre la SUNAT y el Ministerio de Educación.

Este resultado de alguna manera valida lo que señala Vásquez (1993, p.18) que en muchos países emergentes al igual que en el Perú no existe un buen ambiente para el pago de los tributos, debido a que desde la infancia no existe una formación básica que cree una cultura tributaria adecuada a las necesidades del país, desde las escuelas y en los ambientes familiares.

En la mayoría de los casos los jóvenes aprendieron de impuesto en las universidades, confirmando lo propuesto por diversos estudios y la presente investigación en relación a que para que una persona se cree una cultura tributaria adecuada debe iniciar su preparación desde el hogar y la educación básica regular adquiriendo un conjunto de valores específicos de su grupo socio-cultural, así como de la relación que adquiere con las acciones del Estado mediante el gobierno de turno.

En conclusión conforme se ha demostrado en la aceptación de la primera hipótesis específica se aprueba la necesidad de corregir parámetros educativos de formación en la conciencia ciudadana con la modificación curricular que incluya material académico que prepare a los educandos en el reconocimiento social del contribuyente responsable.

Sin embargo en la no aceptación de la segunda hipótesis especifica relacionada a la pertinencia de contar con un convenio entre la SUNAT y el MINEDU para validar y lograr que los estudiantes adquieran la conciencia necesaria que todo contribuyente debe mantener y con ello el desarrollo de una cultura tributaria propicia a los intereses sociales del país, el resultado es contradictorio pues no se reconoce como premisa la intervención del Estado necesariamente para proyectar cambios en la currícula educativa a nivel básico regular, más aún si este cuenta desde ya con más de diez años de suscrita y a la fecha casi no ha sido implementada.

\section{DISCUSIÓN}

En el Perú el problema de la evasión fiscal se ha caracterizado por ser un problema de cultura que desde hace varios años ha venido siendo tratado para así disminuir la evasión del pago de los tributos. Para una formación tributaria adecuada se presenta la "información" como una herramienta primordial para alcanzar los objetivos satisfactorios en el proceso de motivación y divulgación, lo que permitirá la formación de ciudadanos. 
En este sentido la cultura tributaria es sumamente importante ya que define el comportamiento de los contribuyentes respecto al cumplimiento de sus obligaciones y derechos ciudadanos en materia tributaria. Esto es crear una conciencia tributaria que se materialice con el cumplimiento tributario de sus obligaciones con cero evasión y elusión y mucho menos defraudación al Estado lo que de alguna manera frena el crecimiento y desarrollo de un país.

Como ha sido señalada por diversos autores: "Por lo tanto la educación tributaria de ninguna manera puede reducirse a la enseñanza de prácticas que solamente capaciten para atender los requerimientos del régimen impositivo, tampoco puede limitarse al ámbito de la formalidad fiscal, el orden legal y las razones de su cumplimiento, sino debe ser, necesariamente, una educación orientada hacia el cambio cultural y la revaloración de lo ético dentro del conjunto social."

Esta situación a pesar de su realidad y aceptación por los actores socio-culturales del país, no ha sido adecuadamente tratada por la principal entidad responsable de su gestión como lo es la SUNAT, quien no ha liderado el proceso de cambio cultural del contribuyente, a fin de superar la exigencia media de pago de los tributos como obligación y no sobre la base de una creación de la conciencia tributaria que el país necesita.

En el año 2013 la SUNAT capacitó a 4,946 docentes de Educación Básica Regular (inicial, primaria y secundaria) de todas las regiones del país. Teniendo como objetivo fortalecer el rol de los docentes como promotores de la cultura tributaria en las aulas escolares donde se forman los futuros ciudadanos contribuyentes de nuestro país. El trinomio hogar-escuela-comunidad, se constituye en el núcleo base del Estado y del país, de lo que aprendido allí dependerá el comportamiento social en colectivo bajo la premisa que cultura tributaria se implanta desde los colegios públicos o privados en inicial, primaria y secundaria. Aquí se presenta un esquema del proyecto que venía implantando la SUNAT en el año 2005-2006.

Gráfico 1: Estrategias para generar conciencia tributaria desarrollada por la SUNAT.

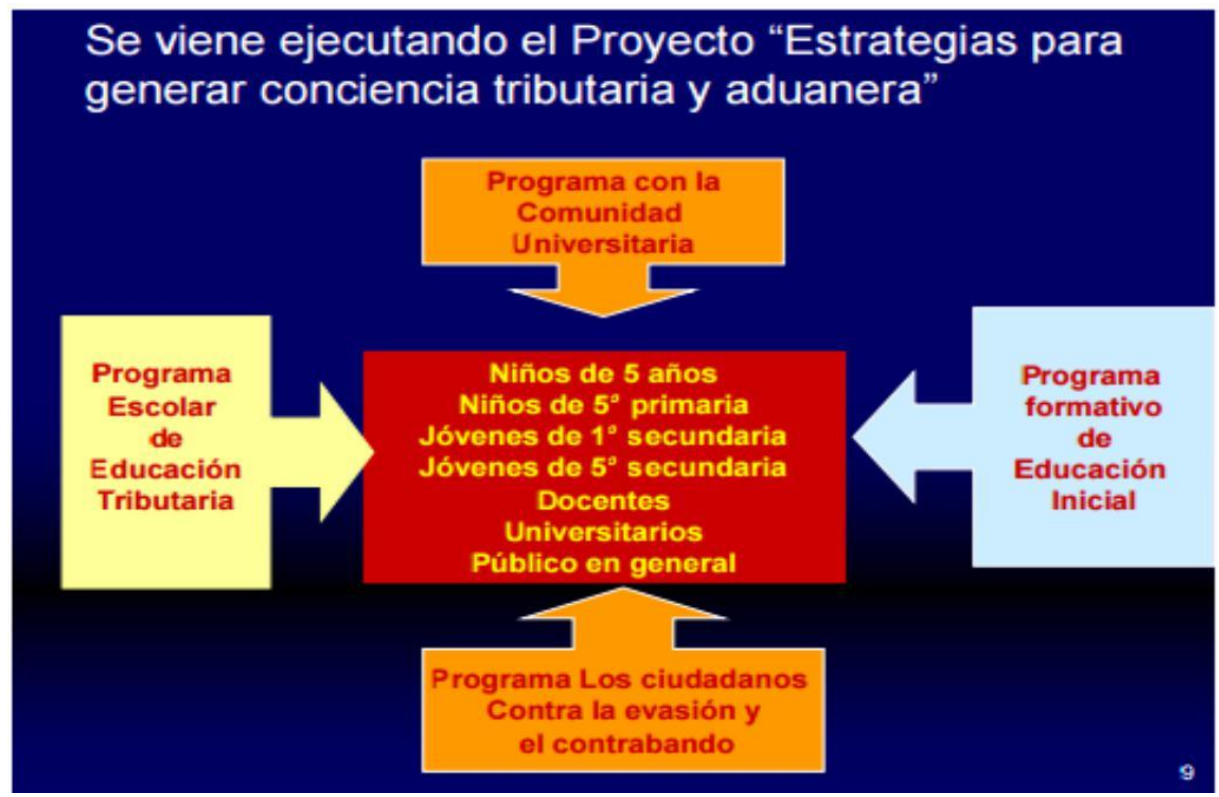


Fuente: SUNAT

\section{REFERENCIAS BIBLIOGRÁFICAS.}

Bravo, F. (2011) Cultura Tributaria. Libro de Consultas (2a ed.). Perú.

Bernal Torres, C. (2010). Metodología de la Investigación (3ra ed.). Colombia: Prentice Hall

Gaona, W. y Tumbaco P., (2009) Tesis: "La educación tributaria como medida para incrementar la recaudación fiscal en Ecuador", rescatada de la página web: ttps://www.dspace.espol.edu.ec/bitstream/123456789/5530/1/D-38880.pdf

Gómez Gallardo, L. M., \& Macedo Buleje, J. C. (2008). La Difusión de la Cultura Tributaria y su Influencia en el Sistema Educativo Peruano.

Hernández Sampieri, R. (2006). Metodología de la Investigación (4ta Edición ed.). México: The McGraw-Hill.

Mogollón Díaz, V. (2014). Tesis de Grado. Nivel de Cultura Tributaria en los Comerciantes de la ciudad de Chiclayo en el Periodo 2012. Chiclayo, Perú: Universidad Católica Santo Toribio de Mogrovejo.

Valencia M. (2016) "La política tributaria y su influencia en la cultura tributaria de los comerciantes del mercado Vinocanchon del Distrito de San Jerónimo - Cusco"

Vásquez J. (abril 1993).Como influir en la conciencia tributaria del Contribuyente para mejorar su comportamiento. En J. Etcheverry (Presidencia). Tendencias modernas de la tributación y la Administración tributaria. Conferencia llevada a cabo en la $27^{\circ}$ Asamblea general del centro Interamericano de Administradores tributarios. Santiago, Chile.

SUNAT. Página web. (www. SUNAT. gob.pe). 